\title{
Super-Heavy Ions Acceleration Driven by Ultrashort Laser Pulses at Ultrahigh Intensity
}

\author{
Pengjie Wang ${ }^{1},{ }^{1}$ Zheng Gong ${ }^{1,}, \|$ Seong Geun Lee, ${ }^{2,3}$ Yinren Shou, ${ }^{1}$ Yixing Geng, ${ }^{1}$ Cheonha Jeon, ${ }^{2}$ I Jong Kim® ${ }^{4}$, \\ Hwang Woon Lee, ${ }^{2}$ Jin Woo Yoon ${ }^{2,5}$ Jae Hee Sung, ${ }^{2,5}$ Seong Ku Lee, ${ }^{2,5}$ Defeng Kong, ${ }^{1}$ Jianbo Liu, ${ }^{1}$ Zhusong Mei®, ${ }^{1}$ \\ Zhengxuan Cao, ${ }^{1}$ Zhuo Pan® ${ }^{1}$ Il Woo Choi, ${ }^{2,5, *}$ Xueqing Yan, ${ }^{1, \dagger}$ Chang Hee Nam®, ${ }^{2,3, \$}$ and Wenjun Ma® ${ }^{1, \S}$ \\ ${ }^{1}$ State Key Laboratory of Nuclear Physics and Technology, School of Physics, CAPT, Peking University, \\ Beijing 100871, China \\ ${ }^{2}$ Center for Relativistic Laser Science, Institute for Basic Science, Gwangju 61005, Korea \\ ${ }^{3}$ Department of Physics and Photon Science, Gwangju Institute of Science and Technology, \\ Gwangju 61005, Korea \\ ${ }^{4}$ Center for Scientific Instrumentation, Korea Basic Science Institute, Daejeon 34133, Korea \\ ${ }^{5}$ Advanced Photonics Research Institute, Gwangju Institute of Science and Technology, \\ Gwangju 61005, Korea
}

(Received 12 September 2020; revised 17 February 2021; accepted 12 April 2021; published 3 June 2021)

\begin{abstract}
The acceleration of super-heavy ions (SHIs, mass number of about 200) from plasmas driven by ultrashort (tens of femtoseconds) laser pulses is a challenging topic awaiting a breakthrough. Detecting and controlling the ionization process and adopting the optimal acceleration scheme are crucial for the generation of highly energetic SHIs. Here, we report the experimental results on the generation of deeply ionized super-heavy ions $\mathrm{(Au}$ ) with unprecedented energy of $1.2 \mathrm{GeV}$ utilizing ultrathin targets and ultrashort laser pulses at an intensity of $10^{22} \mathrm{~W} / \mathrm{cm}^{2}$. A novel self-calibrated diagnostic method was developed to acquire the absolute energy spectra and charge-state distributions of $\mathrm{Au}$ ions abundant at the charge state of $51+$ and extending to $61+$. The measured charge-state distributions supported by $2 \mathrm{D}$ particle-in-cell simulations serve as an additional tool to inspect the ionization dynamics associated with SHI acceleration, revealing that the laser intensity is the crucial parameter over the pulse duration for Au acceleration. Achieving a long acceleration time without sacrificing the strength of the acceleration field by utilizing composite targets can substantially increase the maximum energy of Au ions.
\end{abstract}

DOI: $10.1103 /$ PhysRevX.11.021049

Subject Areas: Plasma Physics

\section{INTRODUCTION}

Laser-driven acceleration of energetic ions is an attractive topic [1-3], owing to its unique features such as an ultrahigh accelerating gradient, micrometer-scale source size, high beam density, and low emittance [4-6]. In particular, laserdriven ion acceleration promises to generate super-heavyion beams with ultrahigh intensity and multiple charge states. Such a super-heavy-ion source is highly desired for numerous applications, including fission-fusion reaction [7] driven by ultrahigh-intensity thorium beams, heavy-ion

\footnotetext{
*iwchoi@gist.ac.kr

†x.yan@pku.edu.cn

*chnam@gist.ac.kr

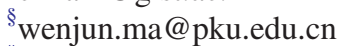

"Present address: Max Planck Institute for Nuclear Physics, Heidelberg 69117, Germany.

Published by the American Physical Society under the terms of the Creative Commons Attribution 4.0 International license. Further distribution of this work must maintain attribution to the author(s) and the published article's title, journal citation, and DOI.
}

accelerators $[8,9]$ that require the injection of multiplecharge-state beams, heavy-ion fusion research [10], and the generation of warm dense matter [11]. Compared with the acceleration of low $-Z$ ions $(80 \mathrm{MeV} /$ nucleon for $\mathrm{C})$ and mid- $Z$ ions (10-20 MeV/nucleon for $\mathrm{Al}^{13}, \mathrm{Fe}^{26}$, and $\mathrm{Ag}^{47}$ ) [4,12-15], the accessible energy of super-heavy ions (SHIs) from laser-plasma interactions is quite low for a long time. Note that $2 \mathrm{MeV} /$ nucleon of $\mathrm{Pb}^{82}[16]$ and $5 \mathrm{MeV} /$ nucleon of $\mathrm{Au}^{79}$ [17] ions were reported by employing hundreds of $J$ long-pulse lasers, which is unfavorable for applications that need a high-repetition rate driven by compact and economic laser systems.

Generally, the acceleration of SHIs would be suppressed by the inevitable contamination attached to the surface of solid targets, where the undesired hydrocarbons are more readily accelerated due to their higher charge-to-mass ratio. Resistive heating [18] and laser heating [19] have been proven as useful methods to remove the contamination and thus facilitate heavy-ion acceleration. However, it is still difficult to efficiently accelerate SHIs because they need to be ionized to high charge states as early as possible to experience a long acceleration time [20]. The accessible intensity of $10^{20} \mathrm{~W} / \mathrm{cm}^{2}$ for picosecond laser pulses, which have been widely utilized for the acceleration of low- $Z$ and 
mid- $Z$ ions, is insufficient to deeply ionize collisionless SHIs. The deep ionization of SHIs relies on the collisional ionization in thermalized plasma, which requires higher laser energy but results in a lower scaling factor of the maximum energy of SHIs with laser energy [21]. On the other hand, state-of-the-art multipetawatt femtosecond lasers have recently been proven capable of delivering an intensity higher than $10^{22} \mathrm{~W} / \mathrm{cm}^{2}$. When such an ultraintense femtosecond laser pulse irradiates an ultrathin target [5], huge ponderomotive force can pile up bulk electrons to build an ultrastrong charge separation field, resulting in deep collisionless ionization [22] and prompt injection [23]. It has been predicted that tens of $\mathrm{GeV}$ of thorium $\left({ }^{232} \mathrm{Th}\right.$ ) ions with very high charge states can be obtained [24] by shooting an ultrathin target at an intensity of $10^{23} \mathrm{~W} / \mathrm{cm}^{2}$ with the upcoming 10-petawatt and exawatt class lasers [25]. However, the proof-of-principle experiment on highly charged super-heavy-ion acceleration, driven by the ultrashort femtosecond laser pulses at such ultrahigh intensity, has not yet been demonstrated.

Here, we experimentally and numerically study the generation of deeply ionized energetic Au ions by using ultrashort femtosecond laser pulses at an intensity of $10^{22} \mathrm{~W} / \mathrm{cm}^{2}$. The energy spectra and the absolute chargestate distributions of Au ions were measured by a novel, self-calibrated, diagnostic method. The charge-state distributions from single- and double-layer targets provide additional information to inspect the ionization and acceleration process. With the help of 2D particle-in-cell (PIC) simulations, the influence of the ionization dynamics on the acceleration process is discussed.

\section{EXPERIMENTAL SETUP AND TARGETS}

The experiments were carried out in a 4-petawatt Ti: sapphire laser facility [26] located at the Center for Relativistic Laser Science (CoReLS). The p-polarized laser pulses were tightly focused onto the targets by an $f / 1.6$ offaxis parabolic mirror with an energy of 14-15 J, as shown in Fig. 1(a). The temporal profile of the pulse was measured with a SPIDER right after the pulse compressor. The fullwidth-at-half-maximum (FWHM) duration was $22 \mathrm{fs}$, as
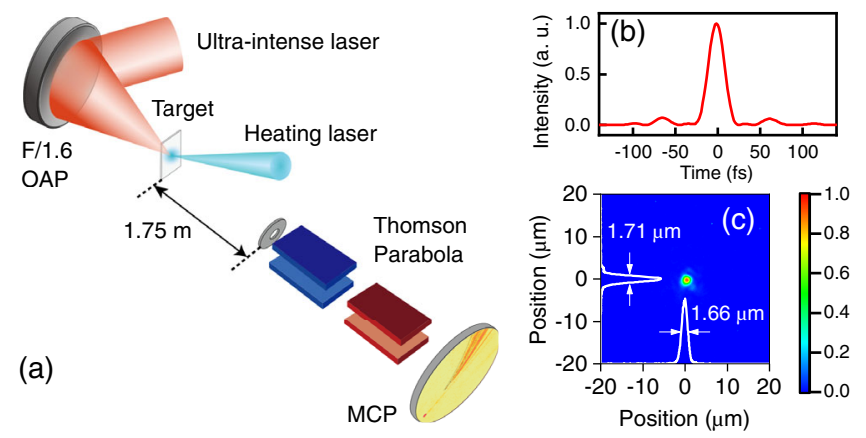

FIG. 1. (a) Experimental setup. (b) Temporal profile and (c) focal spot of driving laser pulses. shown in Fig. 1(b). The focal-spot characterization was performed with a pair of lenses and a 12-bit CMOS camera in the 100-TW mode by attenuating the laser energy with partial reflection mirrors [27]. Figure 1(c) shows the measured focal spot. The best focal-spot size we measured had a near-diffraction-limited size of $1.71 \times 1.66 \mu \mathrm{m}^{2}$ FWHM, and $32 \%$ of the laser energy was concentrated in the FWHM area.

Calculated from the pulse duration and focal-spot measurement, and taking into account the fluctuation in the focal-spot quality, the on-target intensity was $1.1 \pm 0.4 \times$ $10^{22} \mathrm{~W} / \mathrm{cm}^{2}$, which corresponds to a normalized laser amplitude of $a_{0}=e E_{0} / m c \omega \simeq 57-84$, where $e, m, c, \omega$, and $E_{0}$ are electron charge, mass, light speed, laser frequency, and electric field amplitude, respectively. The contrast ratio of the laser pulse was better than $10^{12}$ up to 2 ps before the main pulse by employing a double-plasma mirror system [28], which avoids the prepulse heating and premature expansion of the targets for a low-contrast laser [14].

Single-layer ultrathin foil targets made of $\mathrm{Au}, \mathrm{Ag}, \mathrm{Cu}$, and diamond-like carbon, and double-layer targets composed of carbon nanotube foams (CNF) and ultrathin Au foils were used in the experiments. In contrast to previous work, a water-cooling chemical-vapor-deposition system designed and developed at Peking University enabled the online deposition of CNF onto ultrathin Au foils, which can prevent melting. The density of the CNF was $2.3 \pm 0.5 \mathrm{mg} / \mathrm{cm}^{3}$, corresponding to an electron density of $0.4 \pm 0.1 n_{c}$ when the atoms are fully ionized, where $n_{c}=m \omega^{2} \varepsilon_{0} / e^{2}$ is the critical density of the plasma. The laser pulse was focused onto the CNF side with an incident angle of $2.5^{\circ}$.

A continuous-wave diode laser with a maximum power of $500 \mathrm{~mW}$ was utilized to heat the rear surface of targets to remove the contamination layer before the main laser irradiation. Ions were detected by a Thomson parabola spectrometer (TPS) equipped with a microchannel plate (MCP) with a phosphor. Ions hitting the MCP assembly will produce optical signals, which are imaged by a 16-bit charge-coupled device (CCD) camera. In order to acquire the charge-state distribution of Au ions, the TPS was placed $1.75 \mathrm{~m}$ away from the targets, and the diameter of the collimating tungsten pinhole was $310 \mu \mathrm{m}$, with an ultrasmall-detection solid angle of $2.5 \times 10^{-8}$ sr. Only a few hundred $\mathrm{Au}$ ions can pass the pinhole and arrive at the $\mathrm{MCP}$ per shot. A maximum electric field of $18.2 \mathrm{kV} / \mathrm{cm}$ was applied in TPS to disperse the Au ion Thomson parabolas as much as possible onto the MCP to produce single-ion events.

\section{EXPERIMENTAL RESULTS AND ANALYSIS}

\section{A. Experimental results}

Figures 2(a) and 2(b) show the raw data, measured with TPS, for a 20-nm diamondlike carbon and a double-layer $\mathrm{Au}$ target $(60 \mu \mathrm{m} \mathrm{CNF}+150 \mathrm{~nm} \mathrm{Au})$, respectively. The 

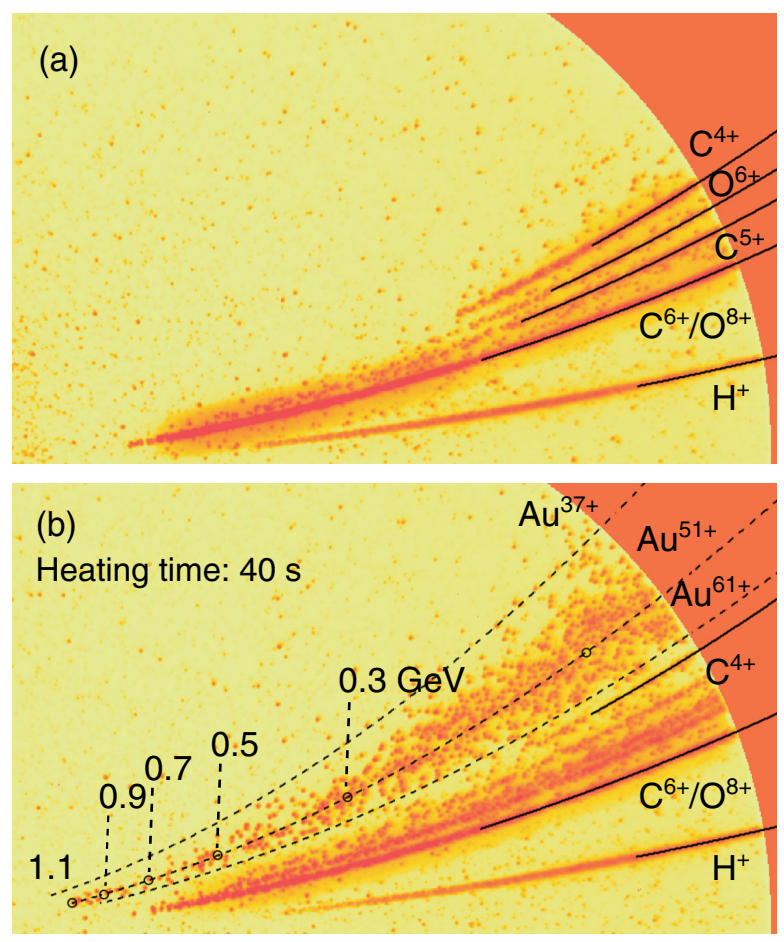

FIG. 2. Raw data measured with TPS from (a) a 20-nm diamondlike carbon target and (b) a double-layer Au target $(60 \mu \mathrm{m} \mathrm{CNF}+150 \mathrm{~nm} \mathrm{Au})$.
TPS-MCP assembly is a common diagnostic tool in laserdriven ion-acceleration experiments. Electric and magnetic fields in TPS can disperse ions in two orthogonal directions, based on the charge-to-mass ratio and energy of ions. Ions with different charge-to-mass ratios form different parabola-like traces on the surface of the MCP. For the diamondlike carbon target, the traces of $\mathrm{H}^{+}, \mathrm{C}^{6+} / \mathrm{O}^{8+}$, $\mathrm{C}^{5+}, \mathrm{O}^{6+}$, and $\mathrm{C}^{4+}$ can be clearly distinguished. $\mathrm{C}$ and $\mathrm{O}$ ions with charge-to-mass ratio of less than $1 / 3$ are not observed, which indicates the acceleration of $\mathrm{C}^{<4+}$ and $\mathrm{O}^{<6+}$ is insignificant at this intensity. Based on this observation, it is inferred that the cluster signals in the region of a charge-tomass ratio of less than $1 / 3$ for double-layer Au targets are caused only by the Au ions. As shown in Fig. 3(a), the clusters have similar shapes and clear boundaries, indicating that they come from single-ion events. We summed the CCD counts for each distinct cluster as the response of a single ion and performed the statistics as a function of the ion energy. The histogram of the responses collected in the region of a charge-to-mass ratio of less than $1 / 3$ from multiple shots is shown in Fig. 3(b). The responses demonstrate a clear dependence on the energy of $\mathrm{Au}$ ions, as depicted in Fig. 3(c). We performed a series of calculations to simulate the MCP response curve, considering the energy, incident angle, and incident positions of the ions (see next subsection). The simulations agree well with the experimental results, confirming each cluster is the signal from a single $\mathrm{Au}$ ion. At the bottom of Fig. 3(b), the responses of the clusters outside the $\mathrm{Au}$ ion region are also presented as the
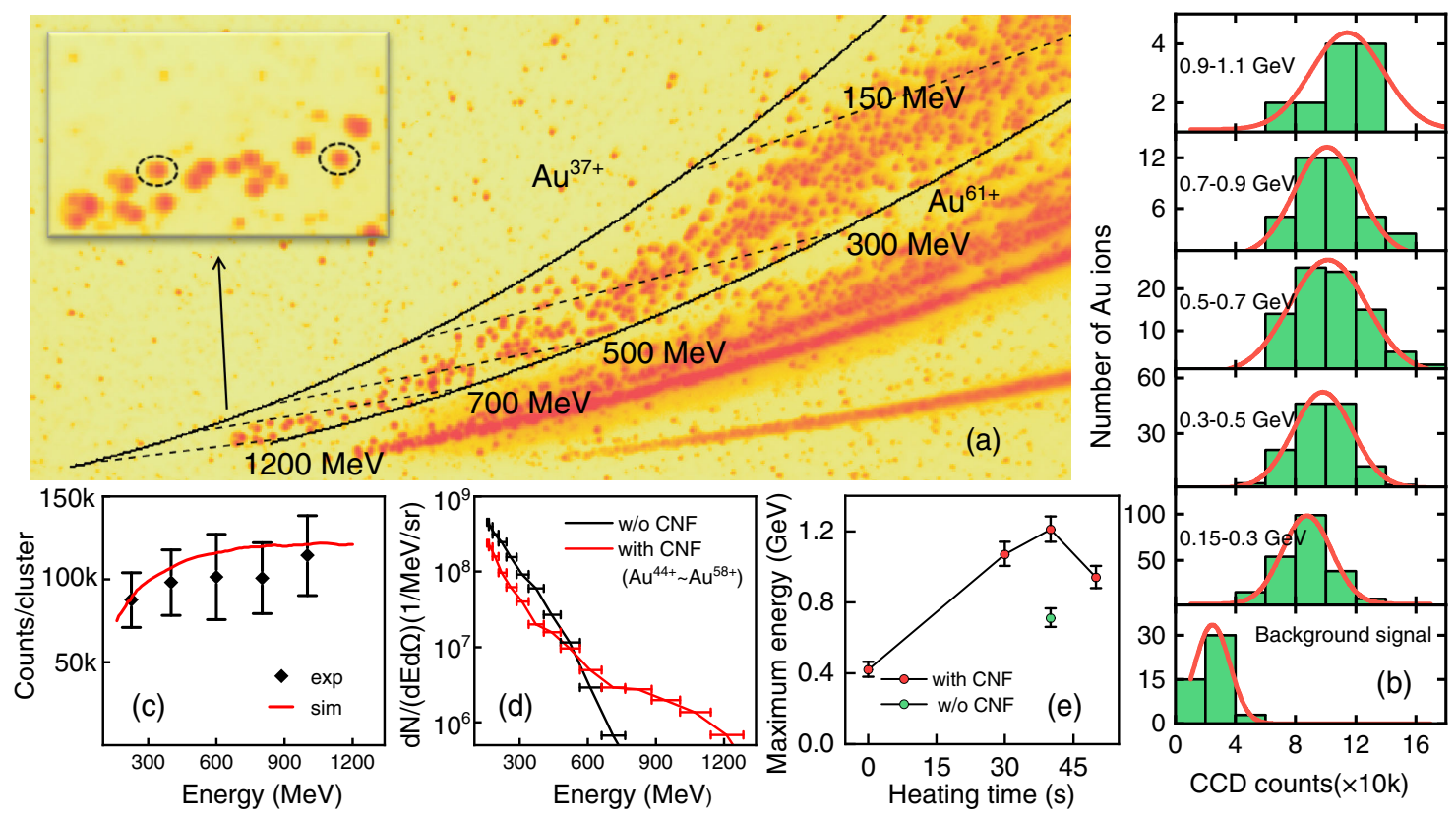

FIG. 3. (a) Schematic of the statistics of single-ion events. The solid lines represent the parabolas of $\mathrm{Au}^{37+}$ and $\mathrm{Au}^{61+}$, and the dashed lines represent the constant energy lines. The inset shows the zoomed clusters of ion signals. (b) Statistical results of single-ion events. The red lines are the Gaussian fitting curves. (c) Responses for Au ions. (d) Energy spectra of Au ions. (e) Maximum energy of Au ions without heating and by varying the heating time. 

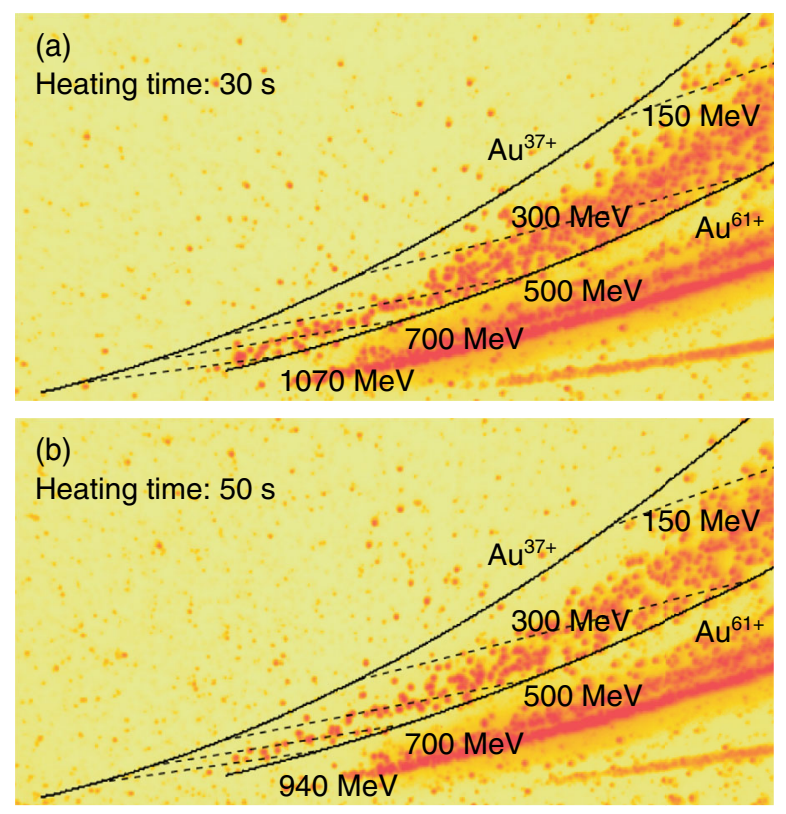

FIG. 4. Raw data measured with TPS from double-layer Au targets $(60 \mu \mathrm{m} \mathrm{CNF}+150 \mathrm{~nm} \mathrm{Au})$ varying heating time of $30 \mathrm{~s}$ (a) and $50 \mathrm{~s}$ (b).

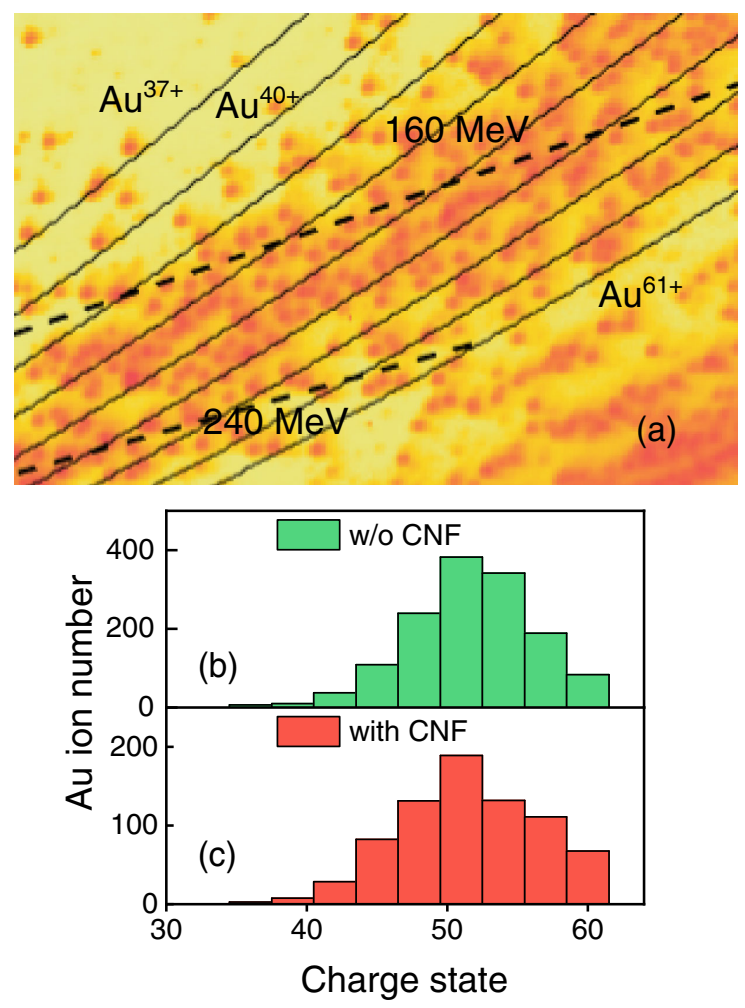

FIG. 5. (a) Schematic of the statistics of the charge-state distributions. The solid lines represent the constant charge states. Charge-state distributions of $\mathrm{Au}$ ions from (b) single-layer and (c) double-layer targets. background signal. They might come from other scattered radiation hitting the MCP, of which counts are significantly lower than that of the Au ions.

Based on the calibration obtained, the absolute energy spectra and charge-state distributions of Au ions can be extracted. Figure 3(d) shows two representative energy spectra. Note that our self-calibrated method can be further confirmed by comparing the order of magnitude from our results with that using CR-39 detectors [17]. The maximum $\mathrm{Au}$ energy from 150-nm single-layer targets is $710 \mathrm{MeV}$ (3.6 MeV/nucleon), 3.6 times higher than the previous results obtained from $14-\mathrm{nm}$ Au foils irradiated by femtosecond lasers [2]. For the double-layer target, the maximum $\mathrm{Au}$ energy is $1.2 \mathrm{GeV}(6.1 \mathrm{MeV} /$ nucleon) at an optimal CNF thickness of $60 \mu \mathrm{m}$, enhanced by 1.7 times compared to the single-layer target. The integrated number of $\mathrm{Au}$ ions with energy greater than $200 \mathrm{MeV}$ is $1.2 \times 10^{10} \mathrm{sr}^{-1}$. We find that the heating time of the targets significantly influences the energy spectra of $\mathrm{Au}$ ions, which is in agreement with previous studies. Figure 3(e) shows the statistics of the maximum Au energy as a function of the heating time. It can be seen that, without heating, the maximum energy of $\mathrm{Au}$ ions is lower than $440 \mathrm{MeV}$. By varying the heating time from $30 \mathrm{~s}$ to $50 \mathrm{~s}$, the maximum energy of Au ions from single-layer and double-layer targets is in the range of $660-710 \mathrm{MeV}$ and $930-1200 \mathrm{MeV}$, respectively. The corresponding raw data of varying heating times are shown in Figs. 4(a) and 4(b).

The absolute charge-state distributions are obtained by counting the $\mathrm{Au}$ ions in striped areas segmented by the constant charge-state lines shown in Fig. 5(a). Technically, all CCD counts in one striped area are summed after subtracting the background and are then divided by the calibrated response of Au ions to obtain the ion numbers in that area. Limited by the resolution of TPS, the statistic division of charge states is set to 3 in Figs. 5(b) and 5(c) for a clear observation of the trend. It is found that there are no significant differences between the cases of single-layer and double-layer targets, which will be analyzed below with the help of numerical simulations. By carefully examining the data, we conclude that a highly charged state of up to 61+ (Ar-like Au ion) is obtained, and the abundant state is $51+$ (Ni-like $\mathrm{Au}$ ion).

\section{B. Simulations confirming the self-calibrated ion responses}

The calibration of the MCP for Au ions in the TPS-MCP assembly has not been reported in the literature. The singleion events, measured here, of the clusters can give a natural self-calibration. In order to confirm such self-calibrations, we simulate response curves for $\mathrm{Au}, \mathrm{Ag}$, and $\mathrm{Cu}$ ions with different energy, and compare them with the measured data in the experiments.

A MCP consists of millions of microchannels made of $10 \mathrm{~s}-\mu \mathrm{m}$-thin conductive glass capillaries. Once an ion 
strikes the inner wall of a channel, multiple secondary electrons are emitted into the channel. Those electrons are accelerated by an electrical field applied along the MCP and may strike the opposite wall in this channel, inducing further electrons repeatedly. Finally, the detected signal can be amplified exponentially. The response of the MCP, for protons and light ions ( $\mathrm{C}$ and $\mathrm{O}$ ), has been calibrated before [29-32].

The theoretical model considered in Refs. [29,32] is used to analyze the $\mathrm{MCP}$ response for $\mathrm{Au}$ ions,

$$
R_{\text {channel }} \propto \frac{1}{\cos \theta}\left(\frac{d E}{d x}\right) \Delta x g
$$

where $\theta$ is the incident angle of ions with respect to the surface of the MCP channel and $(d E / d x)$ is the electronic stopping power of ions striking the surface of the MCP channel one time. Here, $\Delta x$ is the depth above which secondary electrons induced by ions can enter into this channel [33]. Note that $g$ is the gain in this channel, which can be calculated as $g=e^{k\left(L-x_{L}\right) / L} ; k$ is a constant, $L$ is the thickness of the MCP, and $x_{L}$ is the depth from the surface of the MCP, where ions hit the inner surface of the channel. These parameters are marked in Fig. 6(a). Here, we use the MCP (F2226-14P130) from Hamamatsu, with a thickness $(L)$ of $1 \mathrm{~mm}$ and a bias angle of $8^{\circ}$.

For protons with energy greater than $10 \mathrm{MeV}$, the stopping range is larger than the thickness of the wall of the channel. In this case, one proton can pass through more than one channel to generate more electrons. The final response needs to sum up all responses from all the

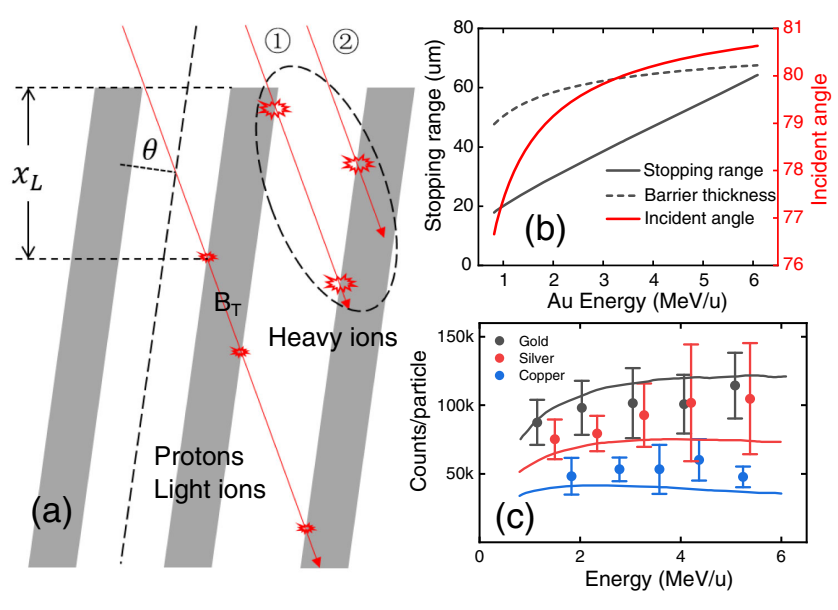

FIG. 6. (a) Schematic of ions interacting with the MCP. The bomb symbols show the generation of secondary electrons on the surface of MCP channels. (b) Stopping range (black solid line) of $\mathrm{Au}$ ions in MCP materials and the barrier thickness (black dashed line) needed to pass through and enter into another channel, depicted as $\mathrm{B}_{\mathrm{T}}$. The incident angle $\theta$ is shown by the red solid line. (c) Responses of heavy ions obtained from experiments (solid dots) and from calculations (solid lines). channels. In our experiment, the energy of $\mathrm{Au}$ ions is lower than $7 \mathrm{MeV} /$ nucleon. Figure 6(b) shows the stopping range and incident angle of an $\mathrm{Au}$ ion into the MCP materials. For $6-\mathrm{MeV} /$ nucleon $\mathrm{Au}$ ions, the maximum stopping range is $64 \mu \mathrm{m}$, which remains smaller than the barrier thickness of the wall along the ion path $\mathrm{B}_{\mathrm{T}}=68 \mu \mathrm{m}$. As a result, most of the Au ions only induce signals from one channel. Nevertheless, there is still a chance that one ion can induce signals from two dots, as we show in case 1 (Response $_{1}$ ) in Fig. 6(a). In case 2 (Response $)_{2}$ ), Au ions hit the channel of the MCP directly, only generating one group of secondary electrons. Therefore, the final response can be written as

Response $=\sum_{i=1}^{2} P_{i} \cdot$ Response $_{i} \propto \frac{1}{\cos \theta}\left(\frac{d E}{d x}\right)_{e} \Delta x \bar{g}$.

The incident angle $\theta$ can be calculated based on the energy and charge state of Au ions, the parameters of TPS, and the bias angle of the MCP. Here, $(d E / d x)_{e}$ can be extracted from SRIM simulations [34], and $\bar{g}$ can be obtained using $\bar{x}_{L}$, the average ion penetration depth inside the channel. Moreover, we give the experimental statistics based on single-ion events, and calculations based on our model, of responses for $\mathrm{Ag}$ and $\mathrm{Cu}$ ions within a similar energy range. The solid dots in Fig. 6(c) show the responses of $\mathrm{Au}, \mathrm{Ag}$, and $\mathrm{Cu}$ ions with respect to their energies obtained in the experiments, and the solid lines are the calculations. According to the Bethe-Bloch equation, the electronic stopping power of ions traversing matter is proportional to the square of the charge state of the incident ion. Therefore, the response for $\mathrm{Au}$ ions would be the largest among these three kinds of ions because of their high energy deposition in the MCP. The theoretical model fits well with the experimental results for three kinds of heavy ions.

\section{PARTICLE-IN-CELL SIMULATIONS AND DISCUSSION}

We performed a series of 2D PIC simulations to illustrate the ionization and acceleration process utilizing the $\mathrm{EPOCH}$ code [35]. The simulation region with a size of $90 \lambda_{0} \times 30 \lambda_{0}$ is uniformly divided into $9000 \times 3000$ mesh grids. A linearly polarized laser with wavelength $\lambda_{0}=0.8 \mu \mathrm{m}$ is incident from the left boundary with FWHM diameter of $1.69 \mu \mathrm{m}$ and FWHM duration of $20 \mathrm{fs}$. A uniform 150-nm Au target with an atom density of $n_{a}=5.96 \times 10^{28} / \mathrm{m}^{3}$ is placed at $0<x<150 \mathrm{~nm}$. In the case of the double-layer target, a near-critical-density plasma slab (representing the CNF) with electron density of $0.4 n_{c}$ is placed ahead of the $\mathrm{Au}$ target. Here, both carbon and Au targets are initialized as neutral atoms [36], which are represented by 20 (C) and $50(\mathrm{Au})$ macroparticles per cell, respectively. The atoms are gradually ionized by the incident laser pulses. The field 

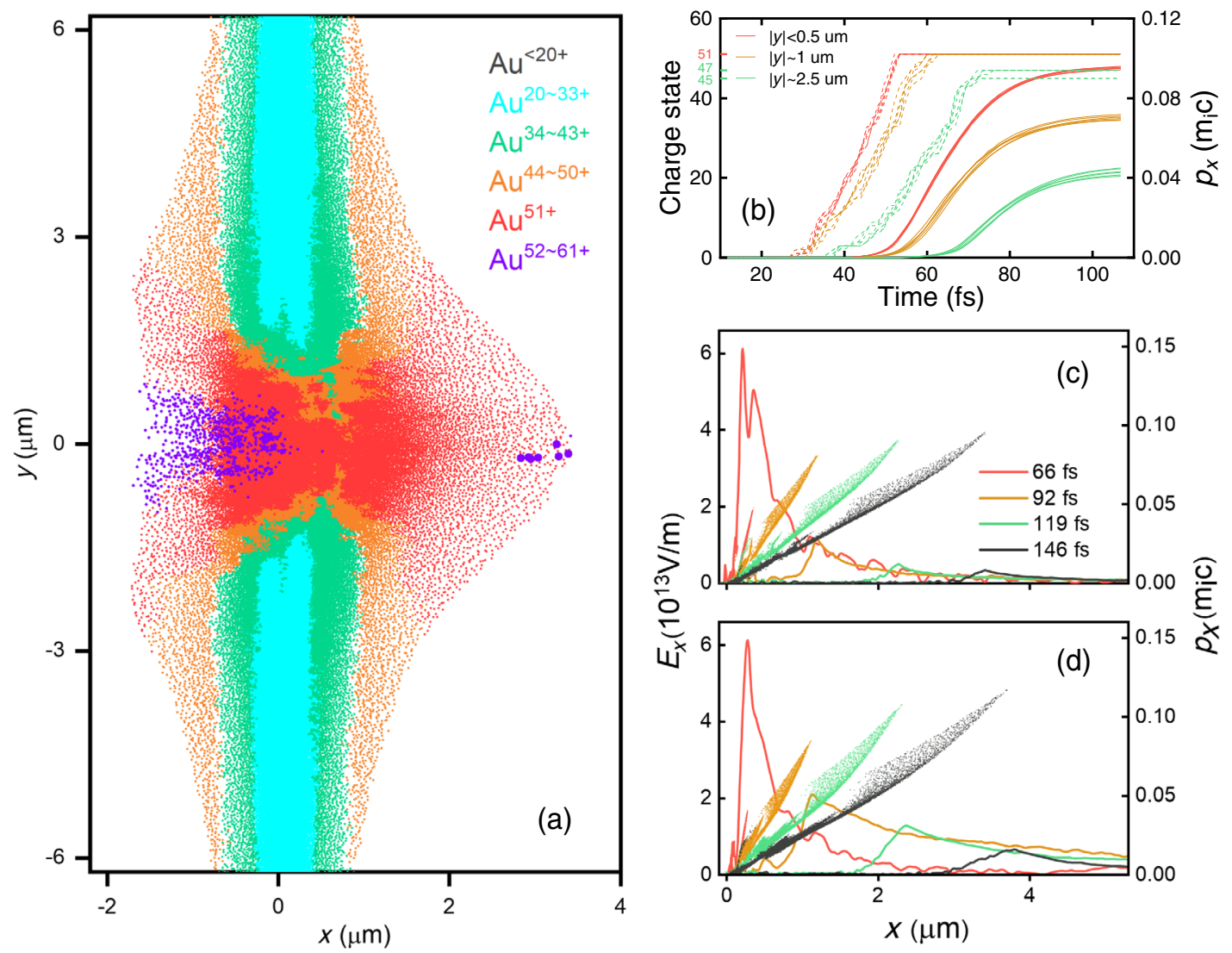

FIG. 7. (a) Spatial distribution of Au ions from a 150-nm single-layer Au target for $a_{0}=60$ at $t=146$ fs. (b) Evolution of the charge states (dashed lines) and the longitudinal momentum $p_{x}$ (solid lines) of Au ions originating from different transverse positions $|y|$ for the target in (a): Each line represents one macroparticle. Evolution of the acceleration field $E_{x}$ (solid lines) and $x-p_{x}$ phase space (scattered dots) for the (c) single-layer target and the (d) double-layer target.

ionization model adopts the Ammasov-Delone-Krainov ionization rate [37], where the equation is averaged over all possible magnetic quantum numbers. The corrected effect from barrier-suppression ionization is incorporated into the Ammasov-Delone-Krainov ionization rate as well [38]. Collisional ionization is not included in the simulations for reasons given below.

We first investigate the influence of the ionization dynamics on $\mathrm{Au}$ acceleration in the single-layer target. The spatial distribution of Au ions at $t=146 \mathrm{fs}$ irradiated by a laser pulse of $a_{0}=60$ is shown in Fig. 7(a). At this moment, the intensity peak of the laser pulse has been reflected for $87 \mathrm{fs}$ from $t=59 \mathrm{fs}$, and the primary acceleration process is completed. Ions near the center of the focal spot have higher charge states than those away from the center as a consequence of the intensity gradient of the driving laser. Judging from their $x$ positions, one can find that $\mathrm{Au}^{\geq 51+}$ are more energetic than $\mathrm{Au}^{<51+}$. They are ionized in the strongest sheath field and accelerated with higher field gradients because of their higher charge-tomass ratio.
The evolution of the charge states and the longitudinal momentum of $\mathrm{Au}$ ions, grouped according to their transverse positions, are examined, as shown in Fig. 7(b). Ions at the center of the focus $(|y|<0.5 \mu \mathrm{m}$, red lines) are first ionized to $\mathrm{Au}^{51+}$ with the maximum ionization rate; they then undergo the longest acceleration time starting from $t=45 \mathrm{fs}$. Ions near the edge of the focal spot $\left(|y| \simeq 1.5 \mu \mathrm{m}\right.$, yellow lines) are ionized to $\mathrm{Au}^{51+} 10 \mathrm{fs}$ later, and their final energy is significantly lower because of the shorter acceleration time. If ions originate further away from the center of the focus $(|y| \sim 2.5 \mu \mathrm{m}$, green lines), they start to ionize and reach their final charge states (45-47) even later, which leads to a more inefficient acceleration as compared to those from the center of the focal spot.

The PIC simulations indicate that the evolution of the acceleration field plays a crucial role in the acceleration of SHIs. As seen from the temporal evolution of $E_{x}$ at $y=0$ for the single-layer and double-layer targets, in Figs. 7(c) and 7(d), respectively, the maximum strengths of $E_{x}$ for the two cases are similar, but the $E_{x}$ of the double-layer target decays much more slowly. The distributions of Au ions in 
$x-p_{x}$ phase space are depicted in Figs. 7(c) and 7(d) as well. At $t=66 \mathrm{fs}$, when the laser peak is reflected while the majority of the super-ponderomotive electrons [15] have not arrived at the second layer, $E_{x}$ acting on Au ions are almost the same for the two cases. However, at $t=92,119$, and $146 \mathrm{fs}$, after the arrival of the superponderomotive electrons, $E_{x}$ exerted on the most energetic ions for the double-layer target are twice as big as that for the single-layer target. Before $E_{x}$ becomes too weak for acceleration, the most energetic Au ions in double-layer targets virtually experience a longer acceleration time in stronger fields, which eventually results in their higher energy.

Instead of employing the double-layer targets, the acceleration time can be prolonged by experimentally stretching the driving pulses as well, which, nevertheless, would lead to the reduction of laser intensity for a given laser energy. For low- $Z$ ions, it was found that the optimal pulse duration is about $100-150$ fs for a given laser as the trade-off between the acceleration time and acceleration gradient [39]. To figure out the dominant factor between the duration and intensity for SHIs acceleration, we carry out a series of simulations by varying the pulse duration from $5 \mathrm{fs}$ to 2 ps while keeping the laser energy constant. Figure 8(a) shows the energy spectra from 150-nm Au targets at different laser intensities. The dependence of the maximum energy of Au ions on the intensity is presented in Fig. 8(b). The results indicate that the laser intensity is more crucial for the acceleration of Au ions over the pulse duration, even when the pulse duration is as short as 5 fs in the case of $a_{0}=120$, which is very different from the result of low- $Z$ ions. The use of double-layer targets leads to the prolongation of the acceleration time without sacrificing the
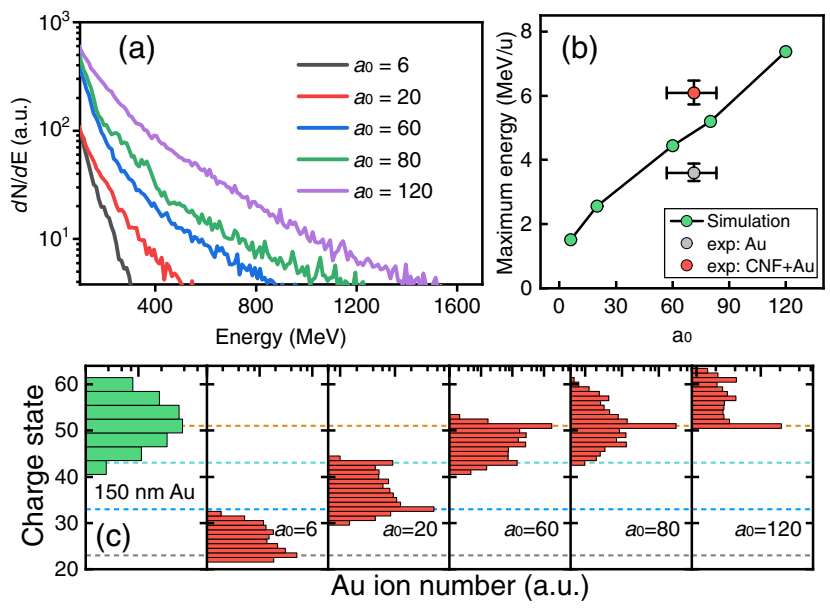

FIG. 8. (a) Energy spectra of Au ions at different intensities controlled by changing the pulse duration while keeping the laser energy constant. (b) Dependence of the maximum Au ion energy on laser intensity. Symbols with error bars mark the experimental results. (c) Charge-state distributions of Au ions in experiments (green bars) and PIC simulations (red bars). acceleration field strength, which is highly favorable for SHIs acceleration.

In the simulations, we find that the charge-state distributions of Au ions are closely related to the laser intensity. Figure 8(c) exhibits the charge-state distributions of $\mathrm{Au}$ ions obtained in the simulations (red bars) at different laser intensities for a 150-nm Au target. The statistics include ions with energy higher than $10 \%$ of the maximum energy and within 10 degrees (half-angle) from the target normal. With the increase of the intensity from $a_{0}=6,20$, to 60, the peak of charge states varies among $\mathrm{Au}^{23+}, \mathrm{Au}^{33+}$, and $\mathrm{Au}^{51+}$, respectively. Such abundant concentration is due to the leap of the ionization energy among the sequential charge states. By comparing the experimental charge-state distributions with simulation ones, an estimation of the acceleration field strength, and furthermore, the on-target laser intensity, can be performed [40]. Figure 8(c) shows that the experimental charge-state distributions elucidate an intermediate state between the simulated results of $a_{0}=60$ and $a_{0}=80$, which is in good agreement with the laser intensity employed in this experiment.

Based on the experimental result of similar charge-state distributions for single-layer and double-layer targets in Figs. 5(b) and 5(c), it is inferred that the maximum acceleration fields in the two cases should be close to each other. Note that the simulation results (red lines, $t=66 \mathrm{fs}$ ) in Figs. 7(c) and 7(d) also confirm this point. This implies that the charge-state distributions of SHIs can be used to probe the ultraintense and transient electric fields, which are very difficult to detect in experiments.

It should be noticed that the highest charge state presented here is $61+($ Ar-like $\mathrm{Au})$, and it is significantly lower than the recently reported value of $72+$ in collisional ionizationdominated laser-plasma interactions at the intensity of $3 \times$ $10^{21} \mathrm{~W} / \mathrm{cm}^{2}$ [41]. We believe this is because the field ionization overwhelms the collisional ionization for ultrathin targets and ultrashort driving pulses at ultrahigh intensity, in our case. The plasma collision rate [42] can be estimated as $\nu_{e e} \sim 10^{-6} n_{e} T_{e}^{-3 / 2} \ln \Lambda_{e e}, \quad \nu_{e i} \sim 10^{-7} Z^{2} \mu^{-1 / 2} n_{i} T_{e}^{-3 / 2} \ln \Lambda_{e i}$, and $\nu_{i i} \sim 10^{-8} Z^{4} \mu^{-1 / 2} n_{i} T_{i}^{-3 / 2} \ln \Lambda_{i i}$. Here, $\nu_{e e}, \nu_{e i}$, and $\nu_{i i}$ are the electron-electron, electron-ion, ion-ion collision rates, respectively; $\mu=m_{i} / m_{p}=197$; and $\ln \Lambda_{e e}, \ln \Lambda_{e i}$, and $\ln \Lambda_{i i}$ are the Coulomb logarithms. In the simulation, we found that the plasma temperature $T_{i}$ and $T_{e}$ in the focal spot quickly increase to more than $1 \mathrm{MeV}$ after the on-target intensity exceeds $3 \times 10^{19} \mathrm{~W} / \mathrm{cm}^{2}$. We find that $\nu_{e e}<$ $10^{-5} \mathrm{fs}^{-1}, \nu_{e i}<10^{-3} \mathrm{fs}^{-1}$, and $\nu_{i i}<10^{-3} \mathrm{fs}^{-1}$ by plugging in the simulation results. Within the interaction timescale of about $100 \mathrm{fs}$, the collision is insignificant in modifying the plasma dynamics.

The collisional ionization may be important if the laser intensity before the main pulse is high and lasts for a long time. We perform collisional 1D PIC simulations (up to $-100 \mathrm{fs}$ ) for a 150 -nm Au target to examine the preionizaiton 

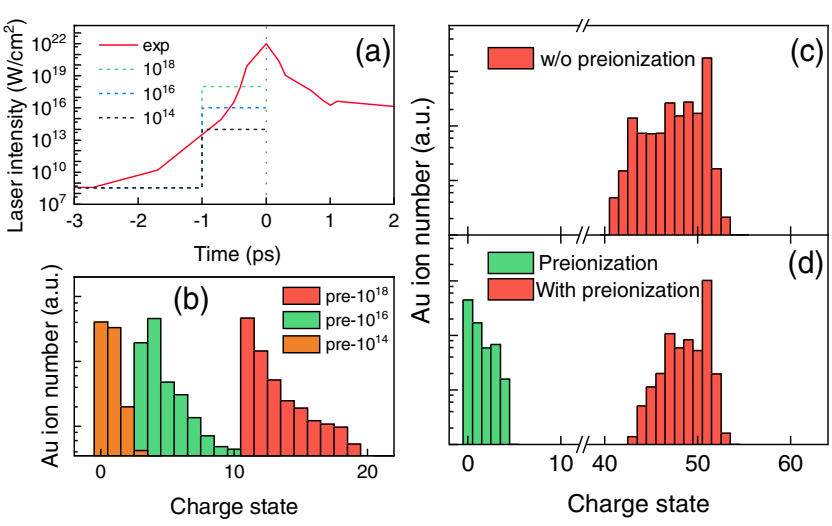

FIG. 9. (a) Temporal profile of the laser pulses after the doubleplasma mirrors (red line), and the assumed 1-ps pedestal with different intensity (dashed lines). (b) Charge-state distribution before the arrival of the main pulse. (c) Final charge-state distribution without preionization. (d) Charge-state distribution before (green bars) and after the main pulse, considering the preionization caused by the rising edge of the laser pulse (red bars).

caused by the rising edge of the laser. The temporal profile [28] of the input pulse measured by a third-order crosscorrelator is shown in Fig. 9(a). As one can see, the use of double-plasma mirrors significantly enhances the contrast, resulting in an intensity of $5 \times 10^{13} \mathrm{~W} / \mathrm{cm}^{2}$ at 1 ps before the main pulse. Figure 9(d) (green bars) shows the charge-state distribution of $\mathrm{Au}$ ions before the arrival of the main pulse. The maximum state is $4+$, and the average state is $0.64+$. To investigate the influence of the preionization on the $\mathrm{Au}$ acceleration, we subsequently perform a 2D simulation with initial charge states obtained from the collisional 1D simulation. The final charge-state distribution, presented in Fig. 9(d) (red bars), has an insignificant difference as compared to that without preionization, as shown in Fig. 9(c), which verifies the opinion that collisionless ionization is dominant in our case. For the integrity of the study, we also perform 1D collisional simulations with a 1-ps pedestal profile at intensities of $10^{14}, 10^{16}$, and $10^{18} \mathrm{~W} / \mathrm{cm}^{2}$, respectively. As shown in Fig. 9(b), the preionization becomes significant for $10^{18} \mathrm{~W} / \mathrm{cm}^{2}$. However, such a bad contrast is inapplicable for ion acceleration, as is well known.

\section{CONCLUSION AND OUTLOOK}

In conclusion, we successfully realized the generation of deeply ionized $\mathrm{Au}$ ions up to $1.2 \mathrm{GeV}$ by using ultrashort femtosecond laser pulses at ultrahigh intensity. The chargestate distribution measurement confirmed by 2D PIC simulations indicates the acceleration fields in near-critical-density double-layer targets have similar maximum strengths compared to those of single-layer targets but decay much slower, which eventually leads to higher $\mathrm{Au}$ energies. The PIC simulations also reveal how the transverse position of Au ions in the focal spot influences their charge states and energy when collisionless ionization is dominant. Unlike low- $Z$ ions and mid- $Z$ ions, the maximum energy of the Au ions predominantly relies on the laser intensity over the pulse duration because of the difficulty in atomic ionization.

This work provides the first experimental results of SHIs acceleration at an intensity of over $10^{22} \mathrm{~W} / \mathrm{cm}^{2}$. The generation of tens of $\mathrm{MeV} /$ nucleon SHIs would be realistically expected by following our scheme at higher intensities. It paves the way to the applications requiring SHI beams with multiple charge states and high density such as injectors for heavy-ion accelerators, heavy-ion fissionfusion reaction, and the generation of warm dense matter.

The self-calibrated diagnosis presented can be used not only to calibrate other heavy ions but also to obtain the absolute charge-state distributions of SHIs in laser-plasma interactions. The measured charge-state distributions can serve as an additional tool to inspect the ionization and acceleration process. Such a method can be used as a universal diagnostic in laser-plasma experiments by doping $\mathrm{Au}$ or other SHIs on the surface of arbitrary targets.

\section{ACKNOWLEDGMENTS}

The authors acknowledge the expertise of the Center for Relativistic Laser Science (CoReLS) staff. The work was supported by the Institute for Basic Science, Korea under the project code IBSR012-D1, the NSFC innovation group project (11921006), the National Grand Instrument Project (2019YFF01014402), Natural Science Foundation of China (Grants No. 11775010, No. 11535001, and No. 61631001), and the Beijing Outstanding Young Scientist Program. The PIC code EPOCH was in part funded by the United Kingdom EPSRC Grants No. EP/G054950/1, No. EP/G056803/1, No. EP/G055165/1, and No. EP/M022463/1. The simulations are supported by the High-Performance Computing Platform of Peking University.

[1] N. Dover, M. Nishiuchi, H. Sakaki, K. Kondo, M. Alkhimova, A. Y. Faenov, M. Hata, N. Iwata, H. Kiriyama, J. Koga et al., Effect of Small Focus on Electron Heating and Proton Acceleration in Ultrarelativistic Laser-Solid Interactions, Phys. Rev. Lett. 124, 084802 (2020).

[2] J. Braenzel, A. Andreev, K. Platonov, M. Klingsporn, L. Ehrentraut, W. Sandner, and M. Schnürer, Coulomb-Driven Energy Boost of Heavy Ions for Laser-Plasma Acceleration, Phys. Rev. Lett. 114, 124801 (2015).

[3] L. Willingale, S. Mangles, P. Nilson, R. Clarke, A. Dangor, M. Kaluza, S. Karsch, K. Lancaster, W. Mori, Z. Najmudin et al., Collimated Multi-MeV Ion Beams from High-Intensity Laser Interactions with Underdense Plasma, Phys. Rev. Lett. 96, 245002 (2006).

[4] M. Nishiuchi, H. Sakaki, T. Z. Esirkepov, K. Nishio, T. Pikuz, A. Y. Faenov, I. Y. Skobelev, R. Orlandi, H. Sako, 
A. Pirozhkov et al., Acceleration of Highly Charged GeV Fe Ions from a Low-Z Substrate by Intense Femtosecond Laser, Phys. Plasmas 22, 033107 (2015).

[5] J. Domański, J. Badziak, and M. Marchwiany, Laser-Driven Acceleration of Heavy Ions at Ultra-relativistic Laser Intensity, Laser Part. Beams 36, 507 (2018).

[6] J. Li, P. Forestier-Colleoni, M. Bailly-Grandvaux, C. McGuffey, A. Arefiev, S. Bulanov, J. Peebles, C. Krauland, A. Hussein, T. Batson et al., Laser-Driven Acceleration of Quasi-Monoenergetic, Near-Collimated Titanium Ions via a Transparency-Enhanced Acceleration Scheme, New J. Phys. 21, 103005 (2019).

[7] D. Habs, P. Thirolf, M. Gross, K. Allinger, J. Bin, A. Henig, D. Kiefer, W. Ma, and J. Schreiber, Introducing the FissionFusion Reaction Process: Using a Laser-Accelerated Th Beam to Produce Neutron-Rich Nuclei Towards the $N=$ 126 Waiting Point of the r Process, Appl. Phys. B 103, 471 (2011).

[8] S. Busold, D. Schumacher, C. Brabetz, D. Jahn, F. Kroll, O. Deppert, U. Schramm, T. E. Cowan, A. Blažević, V. Bagnoud et al., Towards Highest Peak Intensities for UltraShort MeV-Range Ion Bunches, Sci. Rep. 5, 12459 (2015).

[9] P. Ostroumov and K. Shepard, Multiple-Charge Beam Dynamics in an Ion Linac, Phys. Rev. ST Accel. Beams 3, 030101 (2000).

[10] S. Kawata, T. Karino, and A. Ogoyski, Review of Heavy-Ion Inertial Fusion Physics, Mat. Rad. Extremes 1, 89 (2016).

[11] W. Bang, B. Albright, P. Bradley, D. Gautier, S. Palaniyappan, E. Vold, M. S. Cordoba, C. Hamilton, and J. Fernández, Visualization of Expanding Warm Dense Gold and Diamond Heated Rapidly by Laser-Generated Ion Beams, Sci. Rep. 5, 14318 (2015).

[12] D. Jung, L. Yin, D. Gautier, H.-C. Wu, S. Letzring, B. Dromey, R. Shah, S. Palaniyappan, T. Shimada, R. Johnson et al., Laser-Driven $1 \mathrm{GeV}$ Carbon Ions from Preheated Diamond Targets in the Break-out Afterburner Regime, Phys. Plasmas 20, 083103 (2013).

[13] S. Palaniyappan, C. Huang, D. C. Gautier, C. E. Hamilton, M. A. Santiago, C. Kreuzer, A. B. Sefkow, R. C. Shah, and J. C. Fernández, Efficient Quasi-Monoenergetic Ion Beams from Laser-Driven Relativistic Plasmas, Nat. Commun. 6, 10170 (2015).

[14] M. Nishiuchi, N. Dover, M. Hata, H. Sakaki, K. Kondo, H. Lowe, T. Miyahara, H. Kiriyama, J. Koga, N. Iwata et al., Dynamics of Laser-Driven Heavy-Ion Acceleration Clarified by Ion Charge States, Phys. Rev. Research 2, 033081 (2020).

[15] W. Ma, I. J. Kim, J. Yu, I. W. Choi, P. Singh, H. W. Lee, J. H. Sung, S. K. Lee, C. Lin, Q. Liao et al., Laser Acceleration of Highly Energetic Carbon Ions Using a Double-Layer Target Composed of Slightly Underdense Plasma and Ultrathin Foil, Phys. Rev. Lett. 122, 014803 (2019).

[16] E. Clark, K. Krushelnick, M. Zepf, F. Beg, M. Tatarakis, A. Machacek, M. Santala, I. Watts, P. Norreys, and A. Dangor, Energetic Heavy-Ion and Proton Generation from Ultraintense Laser-Plasma Interactions with Solids, Phys. Rev. Lett. 85, 1654 (2000).

[17] F. Lindner, E. McCary, X. Jiao, T. Ostermayr, R. Roycroft, G. Tiwari, B. Hegelich, J. Schreiber, and P. Thirolf, En-Route to the Fission-Fusion Reaction Mechanism:
A Status Update on Laser-Driven Heavy Ion Acceleration, Plasma Phys. Controlled Fusion 61, 055002 (2019).

[18] B. M. Hegelich, B. Albright, J. Cobble, K. Flippo, S. Letzring, M. Paffett, H. Ruhl, J. Schreiber, R. Schulze, and J. Fernández, Laser Acceleration of Quasi-Monoenergetic MeV Ion Beams, Nature (London) 439, 441 (2006).

[19] K. Safronov, S. Gorokhov, V. Flegentov, A. Potapov, D. Gavrilov, A. Kakshin, E. Loboda, and D. Vikhlyaev, LaserDriven Ion Acceleration from Thin Foils Heated by CW Laser, Phys. Plasmas 25, 103114 (2018).

[20] G. Petrov, C. McGuffey, A. Thomas, K. Krushelnick, and F. Beg, Generation of Heavy Ion Beams Using Femtosecond Laser Pulses in the Target Normal Sheath Acceleration and Radiation Pressure Acceleration Regimes, Phys. Plasmas 23, 063108 (2016).

[21] G. Petrov, C. McGuffey, A. Thomas, K. Krushelnick, and F. Beg, Heavy Ion Acceleration in the Radiation Pressure Acceleration and Breakout Afterburner Regimes, Plasma Phys. Controlled Fusion 59, 075003 (2017).

[22] D. Kawahito and Y. Kishimoto, Ionization and Acceleration of Multiply Charged Gold Ions in Solid Film Irradiated by High Intensity Laser, Phys. Plasmas 27, 033108 (2020).

[23] J. Li, A. V. Arefiev, S. S. Bulanov, D. Kawahito, M. BaillyGrandvaux, G. M. Petrov, C. McGuffey, and F. N. Beg, Ionization Injection of Highly-Charged Copper Ions for Laser Driven Acceleration from Ultra-Thin Foils, Sci. Rep. 9, 666 (2019).

[24] J. Domański and J. Badziak, Ultra-Intense Femtosecond Super-Heavy Ion Beams Driven by a Multi-PW Laser, Phys. Lett. A 382, 3412 (2018).

[25] C. N. Danson, C. Haefner, J. Bromage, T. Butcher, J.-C. F. Chanteloup, E. A. Chowdhury, A. Galvanauskas, L. A. Gizzi, J. Hein, D. I. Hillier et al., Petawatt and Exawatt Class Lasers Worldwide, High Power Laser Sci. Eng. 7 (2019).

[26] J. H. Sung, H. W. Lee, J. Y. Yoo, J. W. Yoon, C. W. Lee, J. M. Yang, Y. J. Son, Y. H. Jang, S. K. Lee, and C. H. Nam, 4.2 PW, $20 \mathrm{fs}$ Ti:sapphire Laser at $0.1 \mathrm{~Hz}$, Opt. Lett. 42, 2058 (2017).

[27] J. W. Yoon, C. Jeon, J. Shin, S. K. Lee, H. W. Lee, I. W. Choi, H. T. Kim, J. H. Sung, and C. H. Nam, Achieving the Laser Intensity of $5.5 \times 10^{22} \mathrm{~W} / \mathrm{cm}^{2}$ with a WavefrontCorrected Multi-PW Laser, Opt. Express 27, 20412 (2019).

[28] I. W. Choi, C. Jeon, S. G. Lee, S. Y. Kim, T. Y. Kim, I. J. Kim, H. W. Lee, J. W. Yoon, J. H. Sung, S. K. Lee et al., Highly Efficient Double Plasma Mirror Producing Ultrahigh-Contrast Multi-Petawatt Laser Pulses, Opt. Lett. 45, 6342 (2020).

[29] T. W. Jeong, P. Singh, C. Scullion, H. Ahmed, K. Kakolee, P. Hadjisolomou, A. Alejo, S. Kar, M. Borghesi, and S. Ter-Avetisyan, Experimental Evaluation of the Response of Micro-Channel Plate Detector to Ions with $10 \mathrm{~s}$ of $\mathrm{MeV}$ Energies, Rev. Sci. Instrum. 87, 083301 (2016).

[30] A. Mcllvenny, D. Doria, L. Romagnani, H. Ahmed, P. Martin, S. Williamson, E. Ditter, O. Ettlinger, G. Hicks, P. McKenna et al., Absolute Calibration of Microchannel Plate Detector for Carbon Ions up to $250 \mathrm{MeV}$, J. Instrum. 14, C04002 (2019).

[31] R. Prasad, F. Abicht, M. Borghesi, J. Braenzel, P. Nickles, G. Priebe, M. Schnürer, and S. Ter-Avetisyan, Thomson Spectrometer-Microchannel Plate Assembly Calibration for 
MeV-Range Positive and Negative Ions, and Neutral Atoms, Rev. Sci. Instrum. 84, 053302 (2013).

[32] R. Prasad, D. Doria, S. Ter-Avetisyan, P. Foster, K. Quinn, L. Romagnani, C. Brenner, J. Green, P. Gallegos, M. Streeter et al., Calibration of Thomson Parabola-MCP Assembly for Multi-MeV Ion Spectroscopy, Nucl. Instrum. Methods Phys. Res., Sect. A 623, 712 (2010).

[33] A. M. Then and C. G. Pantano, Formation and Behavior of Surface Layers on Electron Emission Glasses, J. Non-Cryst. Solids 120, 178 (1990).

[34] J. F. Ziegler, M. D. Ziegler, and J. P. Biersack, SRIM-The Stopping and Range of Ions in Matter, Nucl. Instrum. Methods Phys. Res., Sect. B 268, 1818 (2010).

[35] T. Arber, K. Bennett, C. Brady, A. Lawrence-Douglas, M. Ramsay, N. Sircombe, P. Gillies, R. Evans, H. Schmitz, A. Bell et al., Contemporary Particle-in-Cell Approach to Laser-Plasma Modelling, Plasma Phys. Controlled Fusion 57, 113001 (2015).

[36] J. Yu, W. Ma, C. Lin, and X. Yan, Shaping of Ion Energy Spectrum Due to Ionization in Ion Acceleration Driven by an Ultra-Short Pulse Laser, Plasma Phys. Controlled Fusion 60, 115007 (2018).
[37] M. V. Ammosov, Tunnel Ionization of Complex Atoms and of Atomic Ions in an Alternating Electromagnetic Field, Sov. Phys. JETP 64, 1191 (1987).

[38] J. Posthumus, The Dynamics of Small Molecules in Intense Laser Fields, Rep. Prog. Phys. 67, 623 (2004).

[39] J. Schreiber, F. Bell, F. Grüner, U. Schramm, M. Geissler, M. Schnürer, S. Ter-Avetisyan, B. M. Hegelich, J. Cobble, E. Brambrink et al., Analytical Model for Ion Acceleration by High-Intensity Laser Pulses, Phys. Rev. Lett. 97, 045005 (2006).

[40] M. Ciappina, S. Popruzhenko, S. Bulanov, T. Ditmire, G. Korn, and S. Weber, Progress Toward Atomic Diagnostics of Ultrahigh Laser Intensities, Phys. Rev. A 99, 043405 (2019).

[41] R. Hollinger, S. Wang, Y. Wang, A. Moreau, M. Capeluto, H. Song, A. Rockwood, E. Bayarsaikhan, V. Kaymak, A. Pukhov et al., Extreme Ionization of Heavy Atoms in SolidDensity Plasmas by Relativistic Second-Harmonic Laser Pulses, Nat. Photonics 14, 607 (2020).

[42] J. D. Huba, NRL Plasma Formulary (Naval Research Laboratory, 1998), Vol. 6790. 